This is one of a series of $B M J$ summaries of new guidelines based on the best available evidence; they highlight

important recommendations for clinical practice, especially where uncertainty or controversy exists. Further information about the guidance, a list of members of the Guideline Development Group, and an algorithm are in the full version on bmj.com.

${ }^{1}$ Alan Lyell Centre for Dermatology, Western Infirmary, Glasgow, UK

${ }^{2}$ Scottish Intercollegiate Guidelines Network (SIGN), Glasgow

${ }^{3}$ Western Infirmary, Glasgow

${ }^{4}$ Stobhill Hospital, Glasgow

${ }^{5}$ Borders General Hospital,

Melrose, UK

${ }^{6}$ Department of Applied Medicine, University of Aberdeen, Aberdeen, UK ${ }^{7}$ Aberdeen Royal Infirmary, Aberdeen Correspondence to: $\mathrm{MH}$ Hilton Boon michele.hiltonboon@nhs.net

Cite this as: BMJ 2010;341:c5623 doi: 10.1136/bmj.c5623

\title{
GUIDELINES \\ Diagnosis and management of psoriasis and psoriatic arthritis in adults: summary of SIGN guidance
}

\author{
A D Burden, ${ }^{1}$ M Hilton Boon, ${ }^{2}$ J Leman, ${ }^{3} \mathrm{H}$ Wilson, ${ }^{4}$ R Richmond, ${ }^{5}$ A D Ormerod, ${ }^{67}$ on behalf of the Guideline \\ Development Group
}

The degree of disability and negative impact on quality of life caused by psoriasis and psoriatic arthritis are comparable to those of ischaemic heart disease, diabetes, depression, and cancer. ${ }^{1}$ Severe psoriasis and psoriatic arthritis are associated with an increased risk of conditions such as cardiovascular disease, diabetes, and depression. ${ }^{2-5}$ Psoriatic arthritis is underdiagnosed; about a fifth of patients with psoriasis also have psoriatic arthritis. ${ }^{6}$ The management of patients with both conditions may be particularly challenging and require close collaboration among several specialties. This article summarises the most recent recommendations from the Scottish Intercollegiate Guidelines Network (SIGN) on the diagnosis and management of psoriasis and psoriatic arthritis in adults. ${ }^{7}$

\section{Recommendations}

SIGN recommendations are based on systematic reviews of best available evidence. The strength of the evidence is graded as A, B, C, or D (fig 1), but the grading does not reflect the clinical importance of the recommendations. Recommended best practice ("good practice points"), based on the clinical experience of the guideline development group, is also indicated (as GPP).

\section{Diagnosis and assessment in primary care}

- Diagnose chronic plaque psoriasis (psoriasis vulgaris) on the basis of well demarcated bright red plaques covered by adherent silvery white scales affecting any body site, often symmetrically, especially the scalp and extensor surfaces of limbs. The differential diagnosis includes eczema, tinea, lichen planus, and lupus erythematosus (GPP).

- Generalised pustular psoriasis (fig 2) is rare and is characterised by the development of numerous sterile non-follicular pustules within plaques of psoriasis or on red tender skin. This may occur acutely and be associated with fever. The differential diagnosis includes pyogenic infection, vasculitis, and drug eruptions (GPP).
The grade of recommendation relates to the strength of the supporting evidence on which the evidence is based. It does not reflect the clinical importance of the recommendation

- At least one high quality meta-analysis, systematic review of randomised controlled trials, or randomised controlled trial with a very low risk of bias and directly applicable to the target population; or

- A body of evidence consisting principally of well conducted meta-analyses, systematic reviews of randomised controlled trials, or randomised controlled trials with a low risk of bias directly applicable to the target population, and demonstrating overall consistency of results

- A body of evidence including studies rated as high quality systematic reviews of case-control or cohort studies, and high quality case-control or cohort studies with a very low risk of confounding or bias and a high probability that the relation is causal and that are directly applicable to the target population, and with overall consistency of results; or

- Extrapolated evidence from studies described in A

C A body of evidence including well conducted case-control or cohort studies with a low risk of confounding or bias and a moderate probability that the relation is causal and that are directly applicable to the target population and with overall consistency of results; or

- Extrapolated evidence from studies described in B

D Non-analytic studies, such as case reports, case series, expert opinion; or - Extrapolated evidence from studies described in C

Good Practice Points (GPP)

Recommended best practice based on the clinical experience of the guideline development group

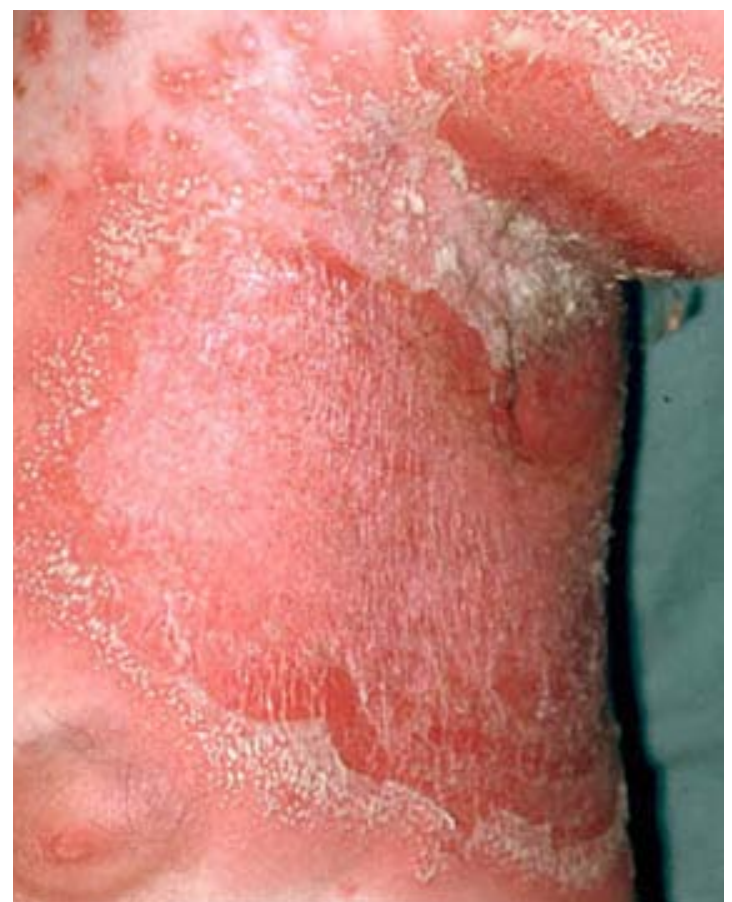

Fig 2 | Generalised pustular psoriasis 


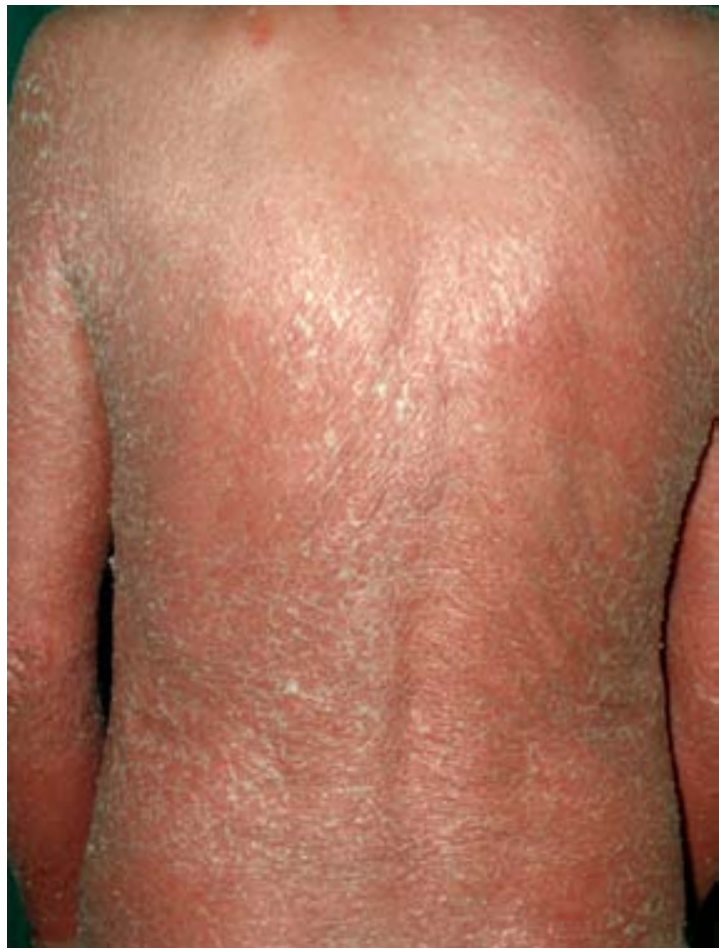

Fig 3 | Erythrodermic psoriasis

- Diagnose guttate psoriasis (fig 4) on the basis of the development over a period of one to seven days of multiple small papules of psoriasis over wide areas of the body. The differential diagnosis includes pityriasis rosea, viral exanthems, and drug eruptions (GPP).

- Consider patient administered screening questionnaires such as the psoriasis epidemiology screening tool (PEST) for early detection of psoriatic arthritis in patients with articular symptoms (C). ${ }^{8}$

- Suspect psoriatic arthritis in patients with psoriasis who develop early morning stiffness lasting more than 30 minutes, spinal stiffness that improves with exercise, joint swelling, joint tenderness, or dactylitis (diffusely swollen or "sausage" digit) (GPP).

- Review patients annually (GPP). As part of the review:

- Optimise topical treatment (see later recommendations) (GPP)

- Document disease severity using the dermatology life quality index (see box outlining online assessment tools) (D)

- Assess for symptoms of arthritis (D)

- Screen for depression (D)

- In patients with severe disease, measure body mass index, blood pressure, and lipid profile, and screen for diabetes mellitus (D).

\section{Referral}

- Offer dermatology referral for patients who fail to respond to topical treatment and who score $>5$ on the dermatology life quality index (D).

- Refer patients with erythrodermic psoriasis (fig 3) or generalised pustular psoriasis to a dermatology department as an emergency (D).

- Refer all patients suspected as having psoriatic arthritis to a rheumatologist for an early diagnosis and appropriate escalation from treatment with disease modifying antirheumatic drugs to treatment with anti-tumour necrosis factor, if required, thereby reducing joint damage (B).

- For guttate psoriasis, consider early referral for consideration of phototherapy in those who fail to respond to topical treatment (GPP).

\section{Treatment}

- Encourage a healthy lifestyle with regular exercise, weight management, cessation of smoking, and moderation of alcohol consumption (D).

- Encourage patients to be actively involved in their care management (D). Discuss treatment options, risks, and benefits with the patient, allowing them more opportunity to be involved in decision making (D).

- To improve concordance, keep the number of treatments a day to a minimum (D).

\section{Topical treatments}

- Consider regular application of emollient to reduce scaling and to help with other symptoms, including itch (GPP).

- In plaque psoriasis, offer short term, intermittent, potent topical corticosteroids or a combined potent corticosteroid plus calcipotriol ointment for rapid improvement (A). Avoid regular use of potent to very potent topical corticosteroids for prolonged periods because of the risk of long term adverse effects, such as cutaneous atrophy (D). For long term use, a vitamin D analogue is appropriate. Choose calcipotriol first, but if this causes troublesome local irritation, switch to an alternative vitamin $\mathrm{D}$ analogue (A). If a vitamin D analogue is ineffective or not tolerated then consider coal tar (solution, cream, or lotion), tazarotene gel, or short contact dithranol (30 minute exposure in patients with a few relatively large plaques of psoriasis) (B).

- In scalp psoriasis, offer short term intermittent use of potent topical corticosteroids or a combination of a potent corticosteroid and a vitamin D analogue (B).

- For patients with thick scaling of the scalp, treat initially with overnight application of salicylic acid,

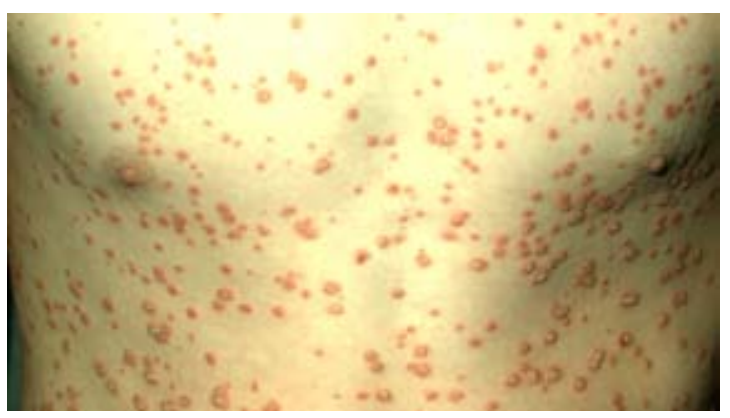

Fig 4 | Guttate psoriasis 


\section{bmj.com archive}

Previous articles in this series

- Rehabilitation of patients with stroke (BMJ 2010;340:C2845)

- Management of obesity (BMJ 2010;340:154)

- Diagnosis and pharmacological management of Parkinson's disease (BM) 2010;340:b5614)

- Diagnosis and management of headache in adults (BMJ 2008;337:a2329)
Online assessment tools for psoriasis and psoriatic arthritis

- Dermatology life quality index (DLQI)-www.dermatology. org.uk/quality/quality-dlqi.html

- Psoriasis area severity index (PASI) calculator-http:// pasi.corti.li/

- Psoriasis epidemiology screening tool (PEST) questionnaire-www.arthritisresearchuk.org/arthritis information/information_for_medical_profes/hands_ on/summer_2009.aspx\#non

- Bath ankylosing spondylitis disease activity index (BASDAl)-http://basdai.com/BASDAl.php

- Bath ankylosing spondylitis functional index (BASFI)http://basdai.com/BASFI.php

tar preparations, or oil preparations (such as olive oil, coconut oil) to remove thick scale (GPP).

- In nail psoriasis, consider topical corticosteroids, salicylic acid, calcipotriol, or tazarotene used alone or in combination (GPP).

- In facial and flexural psoriasis, offer moderate potency topical corticosteroids for first line short term use (B). In patients who fail to respond to this, offer vitamin D analogues or tacrolimus ointment for intermittent use if tolerated (B). Avoid using treatments with irritant properties (dithranol, topical retinoids) in flexural sites (GPP).

\section{Secondary care-psoriatic arthritis}

- Dermatology and rheumatology teams should work closely together to manage patients with severe joint and skin disease (GPP). Aim for monotherapy that treats both skin and joint disease rather than multiple therapies (GPP).

- Offer methotrexate to treat psoriatic arthritis (C), especially when associated with severe cutaneous psoriasis (GPP).

- For patients with peripheral psoriatic arthritis, offer the disease modifying antirheumatic drug leflunomide (A), or sulfasalazine as an alternative (C).

- Offer treatment with a biologic drug (adalimumab, etanercept, or infliximab) to patients with active psoriatic arthritis who have failed to respond to, are intolerant of, or have had contraindications to at least two disease modifying drugs (A).

- Insufficient evidence exists to support a recommendation on the use of ciclosporin in psoriatic arthritis. Patients should not be expected to have failed to respond to ciclosporin before being eligible for treatment with a biologic drug (GPP).

\section{Secondary care-psoriasis}

- For patients with psoriasis who do not respond to topical treatment, offer narrow band ultraviolet B phototherapy (B). For patients who fail this phototherapy, consider psoralen plus ultraviolet A photochemotherapy (B). Advise patients against the use of a sunbed as a source of ultraviolet light for treatment (GPP).
- For patients with severe or refractory psoriasis, consider systemic treatment with ciclosporin, methotrexate, or acitretin after discussion of benefits and risks (B):

- Offer methotrexate for longer term use and where there is concomitant psoriatic arthritis (B)

- Offer ciclosporin for short term intermittent use (A)

- Consider acitretin as an alternative (B) except in women of childbearing potential (GPP).

- For patients who are not suitable for the above systemic treatments, consider fumaric acid esters as an alternative maintenance treatment (B).

- Offer biologic drugs (adalimumab, etanercept, infliximab or ustekinumab) to patients with severe psoriasis who fail to respond to, who have a contraindication to, or are intolerant of phototherapy and other systemic treatments including ciclosporin and methotrexate (A).

- Offer those who have started or switched systemic treatments or biologic drugs the opportunity to enrol on the British Association of Dermatologists Biologics Intervention Register (BADBIR) for assessment of long term relative safety (GPP).

- Ensure that inpatient treatment on a dermatology ward is available for patients with severe psoriasis (D).

- Consider nurse led clinics to deliver services such as follow-up of specialist caseload, repeat access for patients with recurrent disease, and monitoring of systemic treatments (D).

\section{Overcoming barriers}

Resources are needed in primary care for an annual review of patients that fully evaluates the impact of these diseases on quality of life, identifies those patients with more severe disease who require specialist services, and assesses comorbid conditions such as depression and vascular disease. In secondary care, clinical time needs to be made available for multidisciplinary care that optimises patient outcomes over the longer term, in the face of pressures to prioritise new patients. The increased resources required for phototherapy, systemic treatments, biologic treatments, and inpatient treatments are substantial.

Undergraduate and postgraduate medical education needs to reflect the prevalence and importance of psoriasis and psoriatic arthritis in the population. General practitioners may not have received specific rheumatology or dermatology training and consequently may need training in diagnosing, assessing, and managing these conditions. Specialists need to embrace the new concepts around understanding and treating comorbidities of psoriasis and psoriatic arthritis and remain conversant with a rapidly developing therapeutic area.

Contributors: ADB and ADO drafted the article. All authors were involved in the design, appraisal of evidence, analysis of results, revision, and final approval of the article. $\mathrm{ADB}$ is the guarantor

Funding: No funding was received for writing this summary.

Competing interests: All authors have completed the Unified Competing Interest form at www.icmje.org/coi_disclosure.pdf (available on request from the corresponding author) and declare: no funding from any organisation for the submitted work; ADB has been paid for consultancy 
and delivering lectures for Abbott, Pfizer, MSD, Janssen-Cilag, Leo, and Basilea and does consultancy for Merck and Novartis; MHB is employed by SIGN; IL was paid to attend one Abbott Advisory Board meeting and to deliver postgraduate education by Leo, has received meeting expenses from Pfizer, and has undertaken trials funded by Janssen-Cilag, Merck, Serono, Schering-Plough, Abbott, and Pfizer; HW has been paid to provide expert testimony to the Psoriatic Arthritis Advisory Panel and to develop and deliver a Wyeth educational presentation, and has received expenses from Abbott to attend one conference; HW's institution will receive payment for participating in a study of the validity of the PEST questionnaire; RR received travel costs and a conference registration fee from Wyeth; ADO was paid to attend one Abbott advisory board meeting, one Schering-Plough advisory board meeting and to deliver lectures for Wyeth and Abbott, and received expenses to attend one conference from Wyeth and one conference from Janssen-Cilag; ADO's institution has received grants to conduct clinical trials of biologics for Abbott, Wyeth, Schering-Plough, and Janssen-Cilag no other relationships or activities that could appear to have influenced the submitted work.

Provenance and peer review: Commissioned; not externally peer reviewed. Patient consent not required (patient anonymised, dead, or hypothetical).
1 Rapp SR, Feldman SR, Exum ML, Fleischer ABJr., Reboussin DM. Psoriasis causes as much disability as other major medical diseases. J Am Acad Dermatol 1999;41(3 Pt1):401-7.

2 Gelfand JM, Neimann AL, Shin DB, Wang X, Margolis DJ, Troxel AB. Risk of myocardial infarction in patients with psoriasis. JAMA 2006;296:1735-41.

3 Kaye JA, Li L, JickSS. Incidence of risk factors for myocardial infarction and othervascular diseases in patients with psoriasis. Br JDermatol 2008:159:895-902.

4 Neimann AL, Shin DB, Wang X, Margolis DJ, Troxel AB, Gelfand JM Prevalence of cardiovascular risk factors in patients with psoriasis. J $A m$ Acad Dermatol 2006;55:829-35.

5 Kimball AB, Jacobson C, Weiss S, Vreeland MG, WuY. The psychosocial burden of psoriasis. Am J Clin Dermatol 2005;6:383-92.

6 Reich K, Kruger K, Mossner R, Augustin M. Epidemiology and clinical pattern of psoriatic arthritis in Germany: a prospective interdisciplinary epidemiological study of 1511 patients with plaque-type psoriasis. $\mathrm{Br}$ J Dermatol 2009;160:1040-7.

7 Scottish Intercollegiate Guidelines Network (SIGN). Diagnosis and management of psoriasis and psoriatic arthritis (SIGN guideline 121). 2010. www.sign.ac.uk/guidelines/fulltext/121/index.html.

8 Ibrahim G, Buch M, Lawson C, Waxman R, Helliwell PS. Evaluation of an existing screening tool for psoriatic arthritis in people with psoriasis and development of a new instrument: the psoriasis epidemiology screening tool (PEST) questionnaire. Clin Exp Rheumatol 2009;27:469-74.

\title{
PRACTICE POINTER
}

\section{Managing Parkinson’s disease during surgery}

\author{
K A Brennan, ${ }^{1}$ R W Genever ${ }^{2}$
}

${ }^{1}$ Sheffield Teaching Hospitals NHS

Foundation Trust, Sheffield, UK

${ }^{2}$ Chesterfield Royal Hospital

NHS Foundation Trust, Calow,

Chesterfield S445BL, UK

Correspondence to: R W Genever

Richard.Genever@

chesterfieldroyal.nhs.uk

Cite this as: $B M J$ 2010;341:c5718 doi: 10.1136/bmi.c5718

\section{People with Parkinson's disease undergoing surgery are at increased risk owing to their condition and to potential omission of medication. This article looks at what doctors need to consider in this situation and how specialist assistance can be accessed}

Parkinson's disease is a common condition affecting over 100000 people in the UK. ${ }^{1}$ People with Parkinson's disease who undergo surgery have increased mortality and longer hospital stays than people without this condition. ${ }^{2}$ Missing dopaminergic medication during a period of perioperative starvation can result in life threatening complications. ${ }^{34}$ This problem can be further compounded if the absorption of drugs is impaired. ${ }^{5}$ Recognising these hazards is the first step in an approach that may reduce the risk of suboptimal medication faced by people with Parkinson's disease, using three principles.

- Advance planning

- Appropriate prescribing

- Advice from specialists.

\section{Methods}

We reviewed the available literature using PubMed and Google scholar databases up to May 2010. Further papers were identified from the references of articles found by the initial search. We also searched the Cochrane Library. Much of the evidence was based on case reports so selection of papers according to the grade of evidence was not attempted. A study published in July 2010 that added substantial new evidence was included.

\section{Potential complications of missing medication}

The consequences of missing Parkinson's medication can vary enormously. Some people can tolerate a missed tablet without experiencing any major effects. Others become immobile. However, in some situations, missing dopaminergic medication can precipitate a condition known as neuroleptic malignant like syndrome, associated with fever, confusion, raised concentrations of muscle enzyme, and even death. This syndrome is most common in people with more severe Parkinson's symptoms and those on the largest doses of levodopa. ${ }^{6}$

\section{What particular risks are faced?}

Some of the risks relate to Parkinson's disease itself and others to the effects of omitting medication. A retrospective cohort study of 234 people with Parkinson's disease and 40979 controls undergoing major abdominal surgery found a higher incidence of aspiration pneumonia, bacterial infection, and urinary tract infection in the group with Parkinson's. $^{2}$

Case reports have described other perioperative complications in people with Parkinson's disease, including postoperative respiratory failure and postextubation laryngospasm. Other reports mention exacerbation of Parkinson's symptoms during surgery and a case of severe neuroleptic malignant like syndrome precipitated by perioperative starvation for coronary artery bypass grafting. ${ }^{3} 4$

Access to the correct medication at the right time remains a problem for people with Parkinson's disease when they are admitted to hospital. This concern has led to a national awareness campaign from the charity Parkinson's UK. ${ }^{7}$ Lack of awareness of the importance of maintaining medication at the time of surgery places patients at increased risk. 


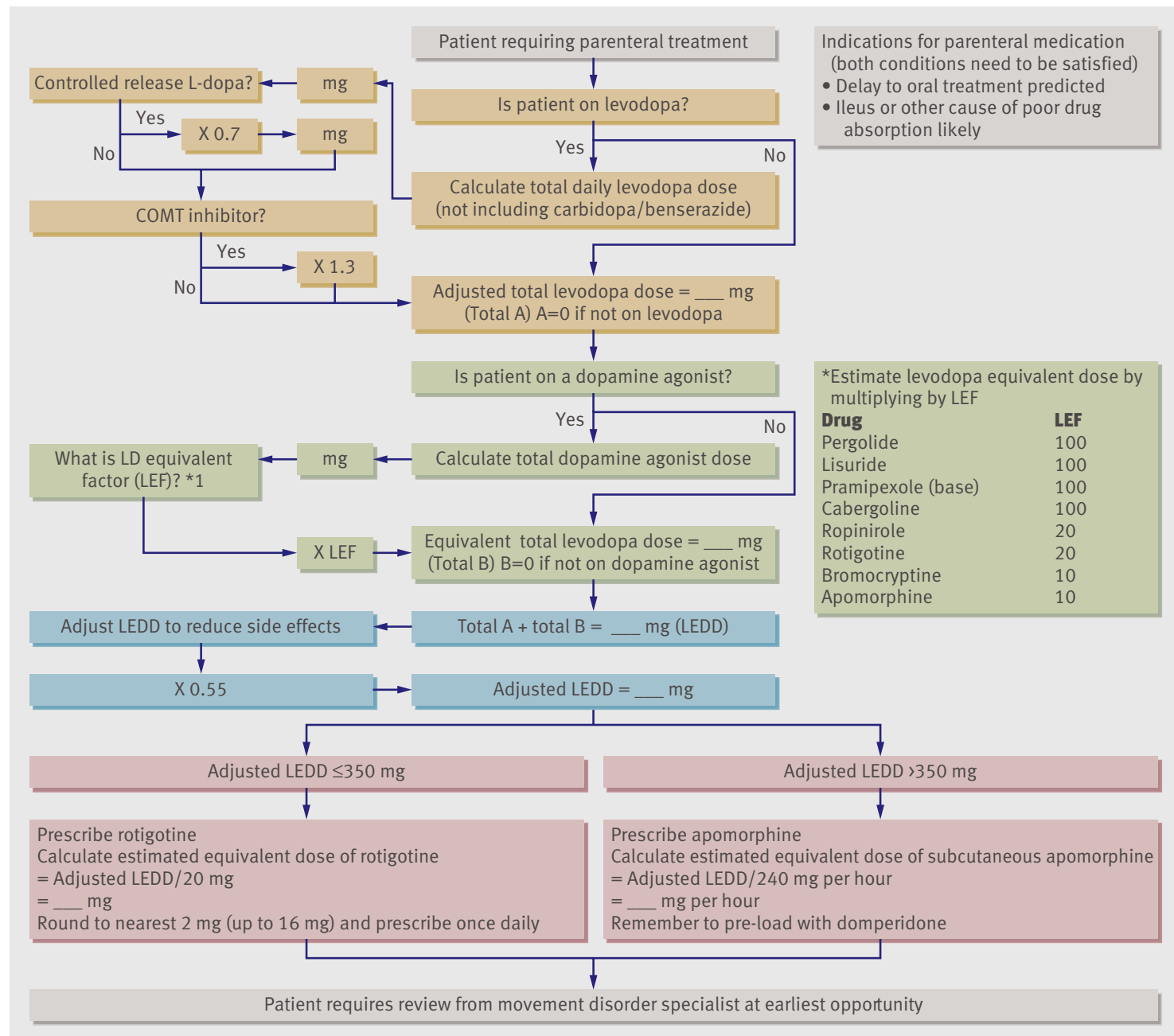

Algorithm for estimating parenteral doses of drugs for Parkinson's disease

\section{Reducing the risks}

Advance planning

As part of a Parkinson's disease education programme, patients should be encouraged to ask that their movement disorder team is consulted before admission. This will enable those at greatest risk to be identified and their treatment individually tailored. The referral could come from the patient's general practitioner, the surgical outpatient department, or the pre-assessment clinic.

The aim is to establish whether the patient's treatment regimen should be altered for the period around their operation and to consider any additional measures that might be preferable.

\section{Who should give the specialist advice?}

Where available, a nurse specialising in Parkinson's disease can play a key part. They may be able to advise directly or discuss the case with the patient's consultant. In areas without specialist nurse support the advice should come directly from the patient's geriatrician or neurologist.

\section{Unplanned surgery and complicated elective surgery}

Specialist preoperative advice cannot always be obtained, particularly for urgent surgery. To allow a treat- ment plan to be enacted at any time, we recommend that hospitals develop a protocol for this situation. We propose a flowchart (figure) to guide a non-specialist until more specific advice can be obtained from the movement disorder team. ${ }^{8}$ Clinicians who are unfamiliar with medication for Parkinson's disease may require assistance from a pharmacist.

\section{Anaesthetic and surgical considerations}

Pre-operative

All patients having surgery under general or regional anaesthetic require preoperative fasting. However, it is recognised that it is safe to continue clear oral fluids up to $2 \mathrm{~h}$ before elective surgery. ${ }^{10}$ It is routine practice to continue oral medications until the time of anaesthetic induction. This practice should be encouraged in patients with Parkinson's. Consideration should be given to placing such patients at the start of the operating list, facilitating greater predictability over the time of fasting and surgery, and ensuring optimum early postoperative disease management.

Induction-regional versus general anaesthesia Several factors affect the choice of anaesthesia. Regional anaesthesia allows monitoring of Parkinson's symp- 
toms and should be considered in people who require very frequent dopaminergic medication. In exceptional circumstances oral medication can be administered intraoperatively, although a case of exacerbation of parkinsonism despite using this approach has been reported. ${ }^{3}$

Some motor symptoms of Parkinson's disease, such as severe dyskinesia, might make a general anaesthetic preferable. The anaesthetist should be aware of the effects of routinely used anaesthetic drugs on parkinsonism. Propofol is commonly used to induce anaesthesia and two case studies have shown that it can exacerbate dyskinesia. ${ }^{11}$ However, propofol may temporarily suppress tremor associated with Parkinson's disease ${ }^{12}$ so it continues to be the first choice of most anaesthetists. Its antiemetic effect may also be beneficial.

The need for intubation should be assessed on an individual basis. Severe Parkinson's disease is associated with excessive salivation that is often due to swallowing dysfunction. If dysphagia is suspected, intubation is the safer choice. In these patients, anticholinergics can increase the viscosity of saliva, further impairing swallowing, and should be used with caution. ${ }^{13}$

\section{Intraoperative}

Antiemetics are routinely given intraoperatively. Centrally acting dopamine antagonists such as prochlorperazine and metoclopramide are likely to exacerbate Parkinson's disease. Domperidone is preferable as it has a largely peripheral mode of action and may be given as a suppository. Other appropriate anti-emetics include 5-hydroxytryptamine-3 antagonists such as ondansetron and the antihistamine cyclizine.

Some patients will have a deep brain stimulator in situ and this should be recorded prominently in the medical notes. Electrocautery may damage the intracranial leads hence the manufacturers recommend its avoidance. However, where necessary, bipolar diathermy should be used. $^{14}$

\section{Post-operative}

An assessment of Parkinson's disease should be made at the earliest opportunity and a judgement made about the patient's ability to absorb enteral medication. A strict nil-by-mouth instruction or active vomiting will mandate an alternative route. The need for parenteral medication is extended in the presence of ileus or delayed gastric emptying. Advice from the Parkinson's disease specialist nurse or consultant should be sought.

\section{Appropriate prescribing}

The overall strategy should be to maintain Parkinson's treatment that closely resembles the patient's usual treatment. Several changes can be recommended, depending on the particular drugs in use and the nature of surgery.

Using a different preparation of the same drug treatment Patients who usually receive levodopa can be given dispersible co-beneldopa via a nasogastric tube during lengthy surgery. A patient with severe Parkinson's disease undergoing liver resection was maintained without appar- ent exacerbation of his condition using this approach. Previous operations had resulted in marked rigidity and inability to swallow secretions in the same patient. ${ }^{15}$

This technique is unsuitable when evidence indicates paralytic ileus. Two cases of worsening parkinsonism have been reported in patients whose recovery was complicated by ileus despite receiving orally disintegrating co-careldopa. ${ }^{5}$

When switching from controlled release levodopa to dispersible co-beneldopa a dose reduction of around $30 \%$ is suggested due to lower bioavailability in controlled release form. ${ }^{16}{ }^{17}$

\section{Using alternative Parkinson's drugs in the} perioperative period

Enteral medication would not be appropriate for a patient in whom post-operative ileus or delayed gastric emptying is expected. The choices for this patient are suboptimal treatment with its associated risks, or a parenteral drug (apomorphine or rotigotine).

Apomorphine is a highly potent dopamine agonist which is delivered subcutaneously. Switching a patient from oral treatment to apomorphine for the duration of surgery would avoid the problems of drug absorption seen with paralytic ileus. Due to its high potency, even patients on large doses of treatment are likely to get effective control of their symptoms from apomorphine. The main disadvantages are emetogenesis (which necessitates concomitant use of domperidone), neuropsychiatric sideeffects such as hallucinations, and hypotension.

Rotigotine is a relatively new agent. It is also a dopamine agonist and is delivered transdermally with a patch. In an open label study, 14 patients were switched from their usual treatment to rotigotine the day before they underwent surgery. The switchover was felt by clinicians and patients to be easy and only one patient had a side effect (transient hallucinations and nausea) that was likely to have been caused by their medication. ${ }^{18}$ Its advantages are ease of use and tolerability but it may not be sufficiently potent to provide adequate treatment for patients on higher dose treatment regimens.

\section{Switching to apomorphine or rotigotine}

A number of articles have proposed levodopa equivalent doses for the various treatments. By using these data and head-to-head studies of rotigotine and ropinirole, it is possible to construct an estimated dose equivalent table for all the currently available drugs (see figure). ${ }^{8}{ }^{9}$ In this situation our practice is to initially prescribe a lower than equivalent dose to reduce the risk of side effects but early review and adjustment of the dose is recommended.

\section{Advice from a specialist}

Even with an agreed advance plan or a written protocol, patients who do not rapidly regain the ability to take their usual Parkinson's medication should be seen by a Parkinson's disease specialist nurse or movement disorder consultant at the earliest opportunity. This allows the treatment plan to be adjusted according to the initial response.

Review of Parkinson's patients by a neurologist following knee replacement has been shown to reduce length of 
stay. ${ }^{19}$ Further research is required to establish whether this benefit can be transferred to Parkinson's patients undergoing different types of surgery.

Contributors: RG initially developed the concept of the paper and led on the review of Parkinson's disease medication research. KB provided the review of anaesthetic papers. Both authors wrote and edited the article jointly. RG is guarantor.

Competing interests: All authors have completed the Unified Competing Interest form at www.icmje.org/coi_disclosure.pdf (available on request from the corresponding author) and declare that $\mathrm{RG}$ has received sponsorship to attend medical conferences from Boehringer Ingelheim, Glaxo Smith Kline, Teva and Lundbeck, and Orion. RG has received honoraria for speaking at meetings from Glaxo Smith Kline and Orion. RG has received honoraria for sitting on advisory boards from Glaxo Smith Kline and Boehringer Ingelheim. KB has no relationships with any companies that might have an interest in the submitted work in the previous 3 years; and RG and $K B$ have no non-financial interests that may be relevant to the submitted work.

Provenance and peer review: Not commissioned, externally peer reviewed.

1 Schrag A, Ben-Schlomo Y, Quinn NP. Cross-sectional prevalence survey of idiopathic Parkinson's disease and parkinsonism in London. BMJ 2000;321:21-2.

2 Pepper PV, Goldstein MK. Postoperative complications in Parkinson's disease. JAGS 1999;47:967-72.

3 Reed AP, Han DG. Intraoperative exacerbation of Parkinson's disease. Anesth Analg 1992;75:850-3.

4 Stoltz M, Thummler D, Schurch M, Renggli I-C, Urwyler A, Pargger H. Fulminant neuroleptic malignant syndrome after perioperative withdrawal of antiParkinsonian medication. BrJAnaesth 2004;96:868-71.

5 Iyer SS, Morgan JC, Sethi KD. Absorption of orally disintegrating levodopa requires intact small bowel function. Neurology 2005;65:1507.

6 Ueda M, Hamamoto M, Nagayama H, Otsubo K, Nito C, Miyazaki T, et al. Susceptibility to neuroleptic malignant syndrome in Parkinson's disease. Neurology 1999;52:777-81.
7 Parkinson's UK. "Get it on time” campaign. www.parkinsons.org.uk/ about_us/policy_and_campaigns-1/campaigns/get_it_on_time_ campaign.aspx

8 Wenzelburger R, Zhang B-R, Pohle S, Klebe S, Lorenz D, Herzog J, et al. Force overflow and levodopa-induced dyskinesias in Parkinson's disease. Brain 2002:125:871-9.

9 LeWitt PA, Boroojerdi B, MacMahon D, Patton J, Jankovic J. Overnight switch from oral dopaminergic agonists to transdermal rotigotine patch in subjects with Parkinson disease. Clin Neuropharmacol 2007;30:256-65.

10 Brady M, Kinn S, Stuart P, Ness V. Preoperative fasting for adults to prevent perioperative complications. Cochrane Database Syst Rev 2009;7:CD005285.

11 Krauss JK, Akeyson EW, Giam P, Jankovic J. Propofol-induced dyskinesias in Parkinson's Disease. Anesth and Analg 1996;83: 420-2.

12 Anderson BJ, Marks PV, Flutter ME. Propofol-contrasting effects in movement disorders. Br/Neurosurg 1994:8:387-8.

13 Nicholson G, Pereira AC, Hall GM. Parkinson's disease and anaesthesia. BrJ Anaesth 2002;89:904-16.

14 Davies RG. Deep brain stimulators and anaesthesia. Br/Anaesth 2005:95:424

15 Furuya R, Hirai A, Andoh T, Kudih I, Okumura F. Successful perioperative management of a patient with parkinson's disease by enteral levodopa administration under propofol anesthesia. Anesthesiology 1998:89:261-3.

16 Sinemet CR and Half Sinemet CR, Summary of product characteristics. Last updated on the eMC 20/11/2009. http:// emc.medicines.org.uk/medicine/9650/SPC/SinemetCR and Half Sinemet CR.

17 Madopar CR. Summary of product characteristics. 2009. http://emc.medicines.org.uk/medicine/1707/SPC/MadoparCR Capsules 125.

18 Wüllner U, Kassubek J, Odin P, Schwarz M, Naumann M, Häck H-J, et al Transdermal rotigotine for the perioperative management of Parkinson's disease. J Neural Trasn 2010;117:855-9.

19 Mehta S, Vankleunen JP, Booth RE, Lotke PA, Lonner JH. Total knee arthroplasty in patients with Parkinson's disease: impact of early postoperative neurologic intervention. Am J Orthop 2008;37:513-6. Accepted: 16 September 2010

ANSWERS TO ENDGAMES, p 1001. For long answers go to the Education channel on bmj.com

\section{PICTURE QUIZ}

\section{A 35 year old smoker with shortness of breath}

1 Multiple cysts, ground glass opacities, and fibrotic changes are the main abnormalities seen on these images.

2 The differential diagnoses include smoking related interstitial lung diseases (desquamative interstitial pneumonia, respiratory bronchiolitis-interstitial lung disease, pulmonary Langherhans' cell histiocytosis), sporadic lymphangioleiomyomatosis, idiopathic interstitial pneumonias (atypical form of idiopathic pulmonary fibrosis, fibrotic form of non-specific interstitial pneumonia), and chronic hypersensitivity pneumonia.

3 Bronchoalveolar lavage and a differential cell count on the fluid obtained (to look for lymphocytosis, pigmented macrophages, and CD-1a cells). A surgical lung biopsy should be performed when the images on high resolution computed tomography are not typical of a specific interstitial lung disease, as in this case. The biopsy showed areas of subpleural fibrosis as well as bronchocentric fibrosis with occasional fibroblastic foci.

4 The consensus after the multidisciplinary approach was that the diagnosis was chronic hypersensitivity pneumonia as a result of misuse of cocaine.

5 Drug discontinuation is essential. Although the use of steroids is controversial, methylprednisolone and cyclophosphamide were used to treat pulmonary fibrosis.

\section{STATISTICAL QUESTION}

\section{The Normal distribution}

Answers $a, b, c$, and $d$ are all true.
ON EXAMINATION OUIZ

\section{A patient with splenomegaly}

Answer $D$ is correct.

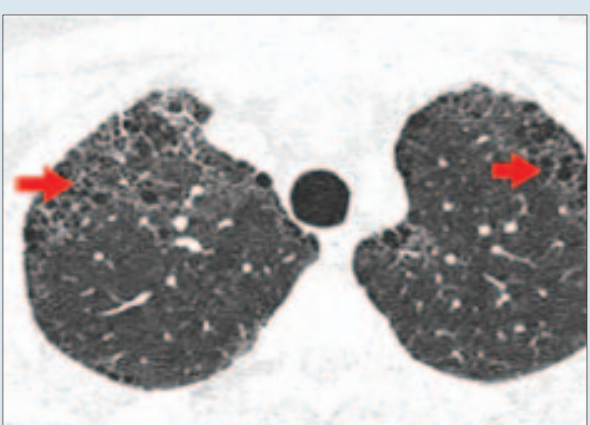

Fig 1 | High resolution computed tomography of the chest showing multiple cysts in the upper lung zones

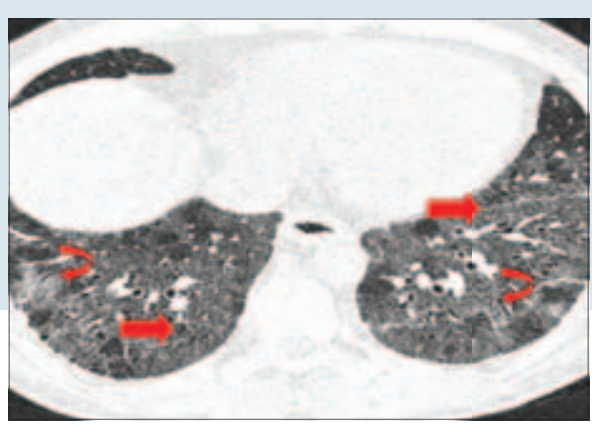

Fig 2 High resolution computed tomography of the chest showing ground glass opacities and fibrotic changes in the lower lung zones 